Article

\title{
Dual-View Integral Imaging 3D Display Based on Multiplexed Lens-Array Holographic Optical Element
}

\author{
Hanle Zhang ${ }^{1,2}$, Huan Deng ${ }^{2, *}$, Minyang $\mathrm{He}^{1,2}$, Dahai $\mathrm{Li}^{2}$ and Qionghua Wang ${ }^{1, *}$ \\ 1 School of Instrumentation and Optoelectronic Engineering, Beihang University, Beijing 100191, China \\ 2 School of Electronics and Information Engineering, Sichuan University, Chengdu 610065, China \\ * Correspondence: huandeng@scu.edu.cn (H.D.); qionghua@buaa.edu.cn (Q.W.)
}

Received: 16 August 2019; Accepted: 11 September 2019; Published: 13 September 2019

Featured Application: In this paper, the dual-view integral imaging 3D display can be used in head-up display, augmented reality and so on.

\begin{abstract}
We propose a dual-view integral imaging 3D display based on a multiplexed lens-array holographic optical element (MHOE). A MHOE is a volume holographic optical element obtained by multiplexing technology, which can be used for dual-view integral imaging 3D display due to the angle selectivity of the volume HOE. In the fabrication of the MHOE, two spherical wavefront arrays with different incident angles are recorded using photopolymer material. In the reconstruction, two projectors are used to project the elemental image arrays (EIA) with corresponding angles for two viewing zones. We have developed a prototype of the dual-view integral imaging display. The experimental results demonstrate the correctness of the theory.
\end{abstract}

Keywords: dual-view display; 3D display; integral imaging; lens-array holographic optical element

\section{Introduction}

Dual-view display provides different images for viewers in different directions. It can meet the needs of different viewers at the same time, and it saves a lot of space and cost. It is increasingly commercialized, especially for car navigation, home entertainment and business applications. For example, when a dual-view display is used in an automobile, it can represent navigation information for the driver and entertainment information for the passenger simultaneously. Some dual-view liquid crystal displays have been proposed [1,2], but they can only display 2D images. For automobile applications, optical see-through capacity is needed because the driver needs to see the road while watching the navigation information.

Recently, 3D displays, including holography display [3-5], integral imaging display [6-9], volumetric display $[10,11]$ and so on, have received extensive attention. Integral imaging is considered to be one of the most promising display technologies. It has the advantages of full parallax [12] and continuous viewpoint [13], and it supplies various physiological depth cues, so viewers can freely change the accommodation and convergence without feeling any visual discomfort [14,15]. Integral imaging has a small viewing angle, and the viewing zone is periodically repeated. Therefore, it is suitable for dual-view or multi-view displays. By using some kinds of multiplexing methods, it can present different 3D images in different directions, and meet the needs of different viewers. In recent years, our team proposed several ways to implement dual-view 3D displays [16-18]. We proposed a dual-view integral imaging 3D display [16], but the viewing angle is only half of the conventional one. In order to increase the viewing angle of the dual-view 3D display, we proposed a dual-view integral imaging 3D display using polarizer parallax barriers [17], but the resolution of each 3D image is reduced to half of the conventional one. In order to solve these issues, Seoul University proposed 
a projection-type dual-view 3D display based on integral imaging [19], but the display system is bulky. Then we proposed a dual-view integral imaging 3D display using an orthogonal polarizer array and polarization switcher [18], but the brightness is not high.

In this paper, we propose a dual-view integral imaging 3D display system based on a MHOE. The viewing angle and resolution of 3D images in the dual viewing zones are almost same as the conventional one. The dual-view 3D display system can provide different 3D images in different zones via controlling the projection beams. In Section 2, the fabrication and reconstruction principles of the MHOE are analyzed, and the projection part of the dual-view 3D display system is designed. In Section 3, the different 3D images are displayed in different viewing zones, and the imaging characteristics of the MHOE are analyzed. The principle of the proposed system is verified by the display experiments.

\section{Structure and Principle}

The schematic diagram of the dual-view integral imaging 3D display system by using MHOE is shown in Figure 1, which consists of an MHOE, projector I and II. The MHOE is used as a projection screen, which records the wavefronts of a micro-lens array from two different directions by using two-step fabrication methods. Projection beams I are projected by projector I, which contain elemental image array (EIA) I, and projection beams I are projected on the MHOE with a pair of angles $\theta_{2}$ and $\theta_{3}$, which satisfies the Bragg condition. Then, 3D images I are reconstructed in the left viewing zone. Projection beams II with EIA II are projected by projector II, and projection beams II are incident onto the MHOE with a pair of angles $\theta_{5}$ and $\theta_{6}$. Then the Bragg condition is satisfied and 3D images II are reconstructed in the right viewing zone. Finally, two different 3D images are reconstructed in the dual viewing zones, respectively.

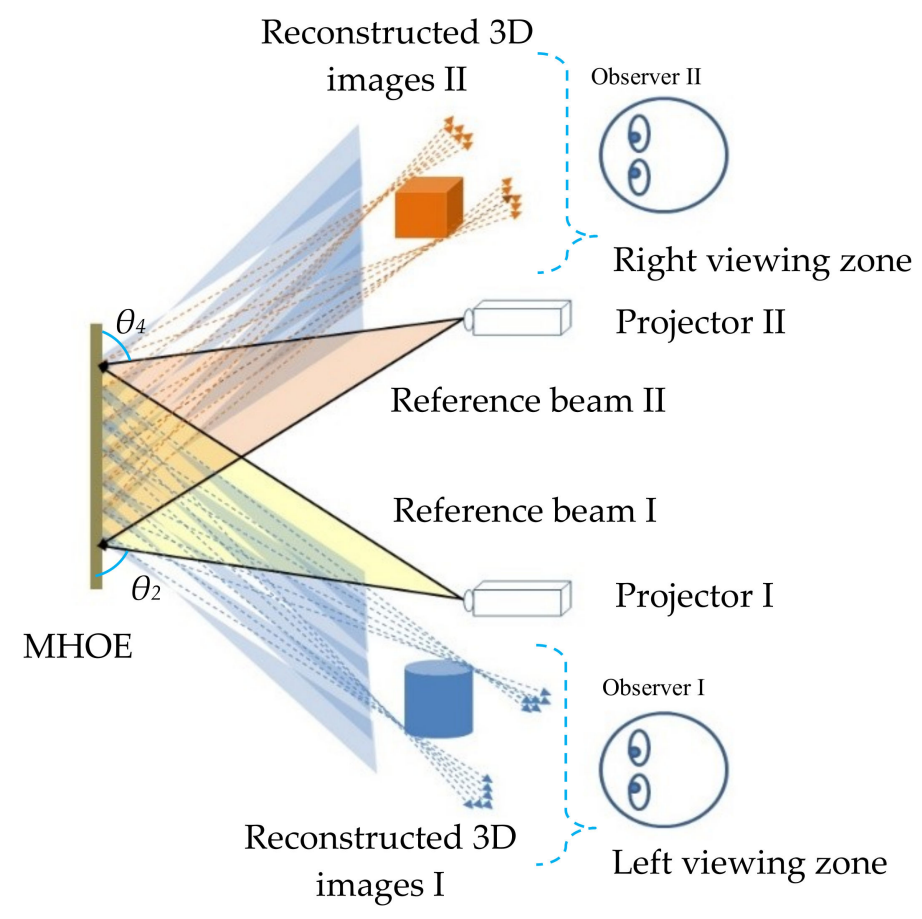

Figure 1. Schematic diagram of the dual-view 3D display system by using the MHOE.

\subsection{Fabrication and Reconstruction Principles of the $M H O E$}

Compared with the conventional holographic optical element (HOE), the MHOE is recorded on the volume hologram, which has the characteristics of angular and spatial multiplexing. HOE has angular selectivity; when the incident beam deviates from the Bragg condition, the diffraction efficiency of the reconstructed beam is reduced. When the incident beam greatly deviates from Bragg condition, 
the diffracted beam cannot be reconstructed. Therefore, multiple optical elements can be recorded on a single holographic plate by angular and spatial multiplexing.

The angular and spatial multiplexing method are used in the fabrication process. Two sets of micro-lens array wavefronts are recorded in a single green sensitive photopolymer material with two different angles. The fabrication process is divided into two steps, which are shown in Figure 2. In the first step, the parallel beam I is incident into the micro-lens array at an oblique angle $\theta_{1}$, and then the spherical wavefront array I is generated. The reference beam I is generated by point source I. Reference beam I interferes with spherical wavefront array I with a pair of angles $\theta_{2}$ and $\theta_{3}$, as shown in Figure 2a. In the second step, the parallel beam II is incident into the micro-lens array at an oblique angle $\theta_{4}$, and then the spherical wavefront array II is generated. The reference beam II is generated by point source II, and reference beam II interferes with spherical wave array II with a pair of angles $\theta_{5}$ and $\theta_{6}$, as show in Figure $2 \mathrm{~b}$. The parallel beams I and II are symmetric to each other. By strictly controlling the exposure time of the first and second steps in the fabrication process. Finally, the required MHOE is obtained.

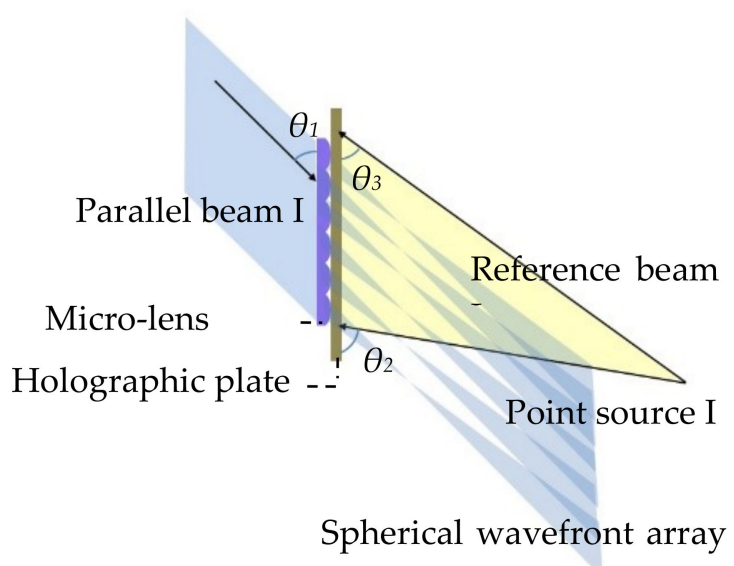

(a)

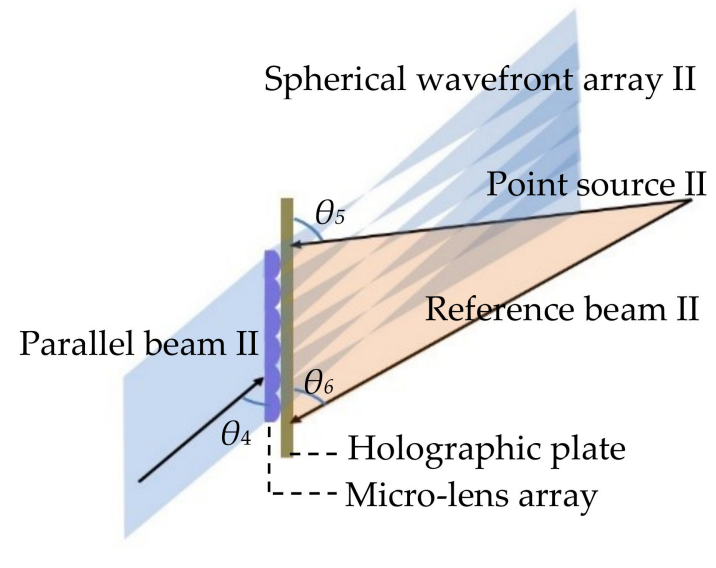

(b)

Figure 2. Fabrication principle of the MHOE, (a) the first step and (b) the second step.

The reconstruction principle of the MHOE is shown in Figure 3. Reference beam I is projected onto the MHOE with a pair of angles $\theta_{2}$ and $\theta_{3}$. The incident angles of the reference beam I are consistent with the angles of fabrication, and the Bragg condition is satisfied. So the spherical wavefront array I can be reconstructed. Reference beam II is also projected onto the MHOE with a pair of angles $\theta_{5}$ and $\theta_{6}$. The incident angles of the reference beam II are the same as the angles of fabrication, and the Bragg condition is also satisfied. Then, the spherical wavefront II is reconstructed. Therefore, the MHOE has the optical characteristics of two sets of micro-lens arrays in different directions. Reference beams I and II are generated by the point source I and II, which have various directional light components. In the reconstruction of 3D images, a Bragg-mismatch occurs when reference beams are produced by projector I and II. So, the angular variation caused by angular deviation of the reconstructed beam should be analyzed.

\subsection{MHOE as Imaging Optical Element in the Dual-View 3D Display}

In the fabrication process, reference beams interfere with the spherical wavefront arrays. The spherical wavefront array consists of many small spherical waves, and the interference fringes are recorded on the holographic material. Each elemental lens in the MHOE is generated by the interference of a large spherical wave (reference beam) and a small spherical wave. The grating vectors of different positions on the MHOE are different. The reference beam is considered as a series of plane beams [20], whose wave vectors $\left(\mathbf{K}_{\mathbf{r} 1 \sim \mathrm{rn}}\right)$ are shown in Figure 4a. The spherical wavefront array can be regarded as a series of small spherical waves, which is shown in Figure 2. Each small spherical 
wave in the spherical wavefront array can also be considered as a series of plane beams, whose wave vectors $\left(\mathbf{K}_{\mathbf{s} \mathbf{~} \sim \mathbf{s n}}\right)$ are shown in Figure 4a. So the change of the reconstructed beam of the MHOE could be explained by the wave vector relationship of the plane beams.

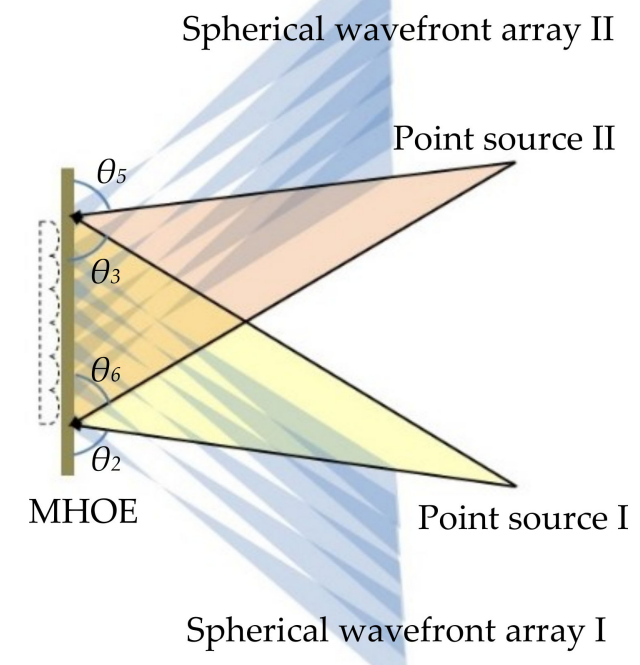

Figure 3. Reconstruction principle of the MHOE.

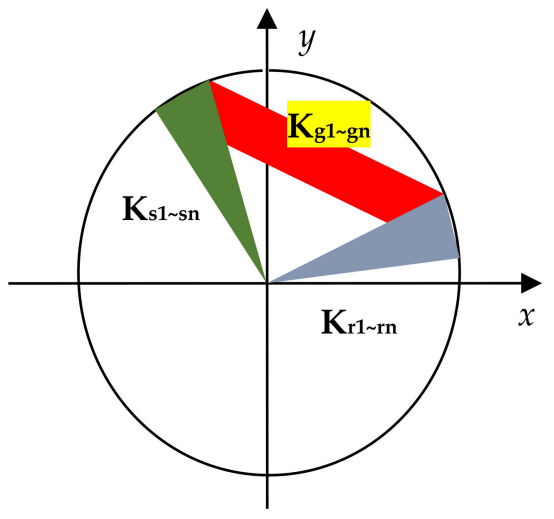

(a)

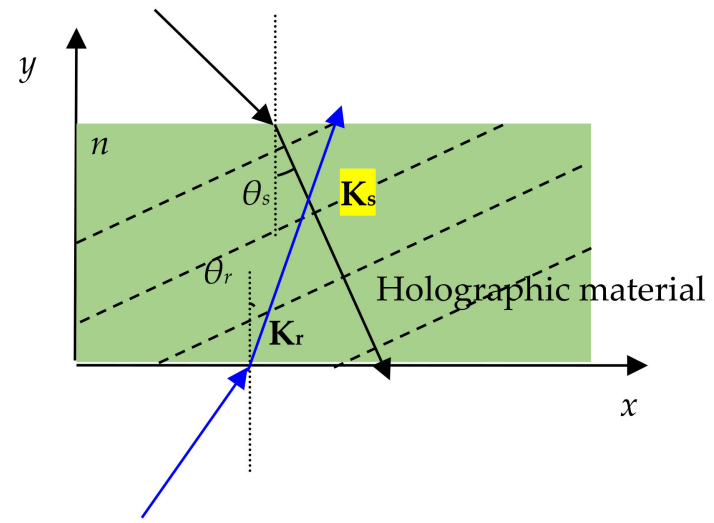

(b)

Figure 4. (a) Grating vector diagram of the elemental lens in the MHOE, and (b) wavefront recording schematic diagram of the elemental lens in the MHOE.

The recorded schematic diagram of the small spherical wave in the MHOE is shown in Figure 4b; the recorded grating vectors $\mathbf{K}_{\mathbf{g}}$ can be expressed as [20]:

$$
\mathbf{K}_{\mathrm{g}}=\vec{x}\left[\left|\mathbf{K}_{\mathrm{s}}\right| \sin \left[\theta_{s}\right]-\left|\mathbf{K}_{\mathrm{r}}\right| \sin \left[\theta_{r}\right]\right]+\vec{y}\left[\left|\mathbf{K}_{\mathrm{s}}\right| \cos \left[\theta_{s}\right]+\left|\mathbf{K}_{\mathrm{r}}\right| \cos \left[\theta_{r}\right]\right]
$$

where $\mathbf{K}_{\mathrm{s}}$ and $\mathbf{K}_{\mathrm{r}}$ are the vectors of the small spherical wave in the spherical wavefront array and reference beam, respectively. In the reconstruction process, the projection beam project onto the MHOE with the vector $\mathbf{K}_{\mathbf{p}}$. The angle of the reconstructed beam can be expressed as [20]:

$$
\theta_{c}=\sin ^{-1}\left[\sin \left[\theta_{p}\right]+\sin \left[\theta_{s}\right]-\sin \left[\theta_{r}\right]\right]
$$

When the projection angle $\theta_{p}$ of the projection beam, the incident angle $\theta_{r}$ of the reference beam and the incident angle $\theta_{s}$ of the small spherical wave incident into the MHOE are determined, the angle 
$\theta_{c}$ of the reconstructed beam changes almost linearly, within the range of the projection angle of the projection beam. Therefore, the MHOE is suitable to be used as the imaging optical element in the dual-view 3D display.

\subsection{Design of Projection Part for the Dual-View 3D Display}

The projection part for the MHOE has to satisfy the Bragg conditions. Projection beam I must satisfy the projection angles $\theta_{2}$ and $\theta_{3}$ for reconstructed 3D images I in the left viewing zone, and projection beam II also must satisfy the projection angles $\theta_{5}$ and $\theta_{6}$ for reconstructed 3D image II in the right viewing zone. In this section, the projection part is realized based on two projectors, so that $3 \mathrm{D}$ image I and II are displayed simultaneously, as depicted in Figure 1.

The principle and design of the projection part for the dual-view 3D display are shown in Figure 5. When reference beam I is projected onto the MHOE with a pair of angles $\theta_{2}$ and $\theta_{3}$, spherical wavefront array I is reconstructed with the angle $\theta_{1}$. When reference beam II is projected onto the MHOE with a pair of angles $\theta_{5}$ and $\theta_{6}$, spherical wavefront array II is reconstructed with the angle $\theta_{4}$. The best view distance of the dual-view 3D display system is $L$, the focal length of the MHOE is $f$ and the pitch is $p$. According to the geometric optics, the viewing angle of the left viewing zone can be expressed as:

$$
\Omega_{1}=\arctan \frac{2 f \tan \theta_{1}}{2 f-p \tan \theta_{1}}-\arctan \frac{2 f \tan \theta_{1}}{2 f+p \tan \theta_{1}}
$$

and the viewing angle of the right viewing zone can be expressed as:

$$
\Omega_{2}=\arctan \frac{2 f \tan \theta_{4}}{2 f-p \tan \theta_{4}}-\arctan \frac{2 f \tan \theta_{4}}{2 f+p \tan \theta_{4}}
$$

The projection part is designed to make sure that the left and right view zone are completely separated, and reconstructed 3D images I and II cannot cause crosstalk, which is shown in Figure 5.

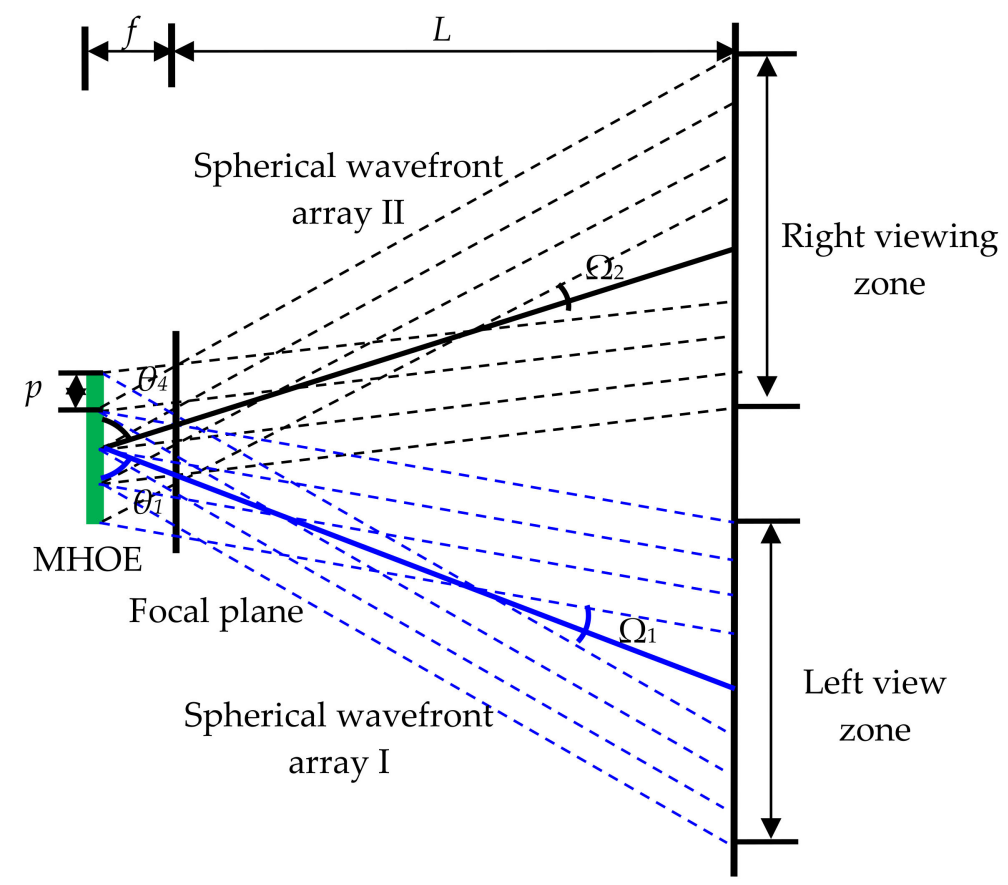

Figure 5. Principle and design of the projection part for the dual-view 3D display. 


\section{Experiments and Results}

\subsection{Fabrication of the $\mathrm{MHOE}$}

In the first step, the experimental setup is shown in Figure 6a. The laser's wavelength and power are $532 \mathrm{~nm}$, and $400 \mathrm{~mW}$, respectively. The thickness, resolution, sensitivity and average refractive index of the photopolymer material are $15 \mathrm{um}, 12,000$ line/mm, $10 \mathrm{~mJ} / \mathrm{cm}^{2}$ and 1.47, respectively. The size of the micro-lens array used to fabricate the MHOE is $150 \mathrm{~mm} \times 150 \mathrm{~mm}$, in which the pith of each lens element is $1 \mathrm{~mm}$ and the focal length is about $3.3 \mathrm{~mm}$. The laser beam is divided into two beams by a beam splitter (BS). One of the laser beams is expanded by a spatial filter (SF) and a collimating lens (CL), then the laser beam incident into the micro-lens array with the angle $\theta_{1}=50^{\circ}$ forms spherical wavefront array I. Another laser beam is reflected by mirror 1 (M1) and mirror 2 (M2), and then passes through the divergent lens (DL) to form spherical wave I. The angles of spherical wave I incident into the holographic plate is controlled to be $\theta_{2}=61^{\circ}$, and $\theta_{3}=81^{\circ}$. The interference occurs between the spherical waves I and spherical wavefront array $\mathrm{I}$, and the interference fringes are recorded on the holographic plate.

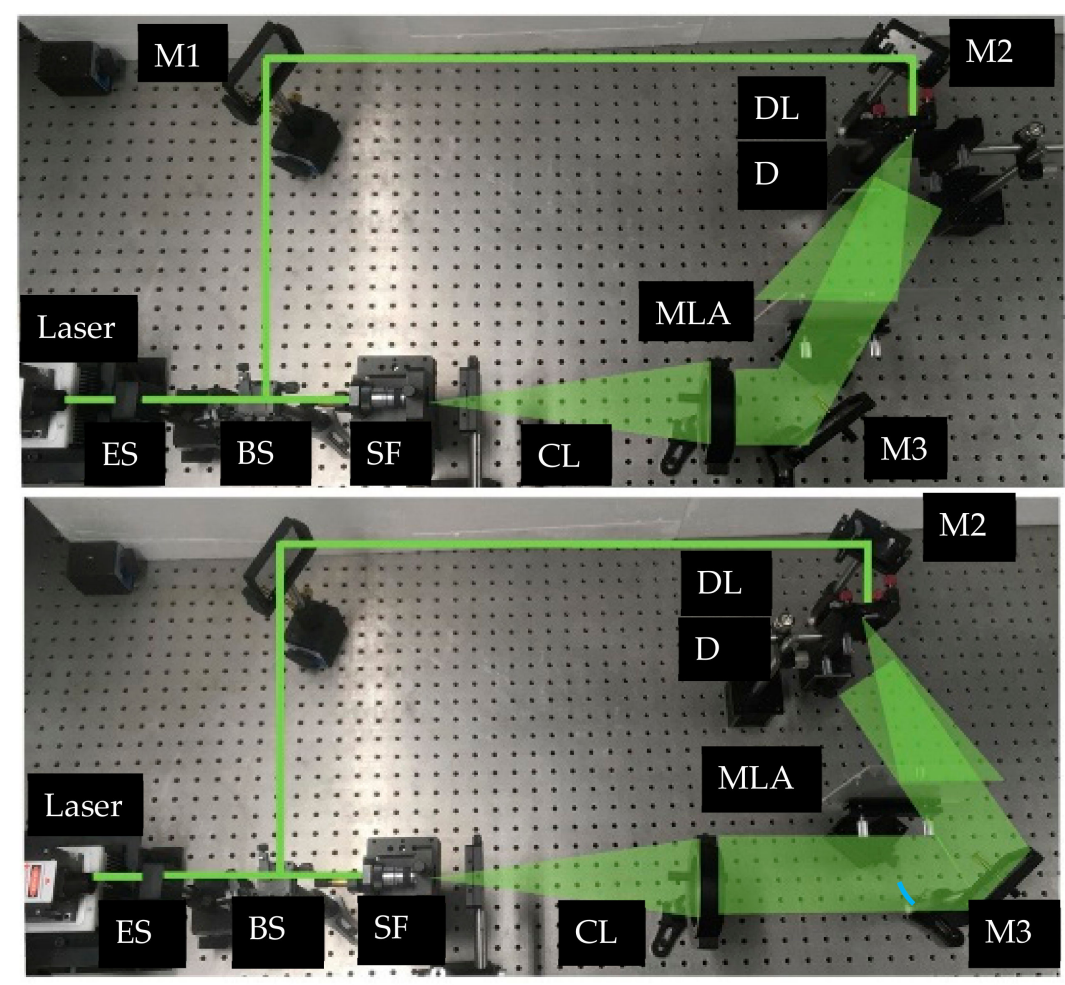

(a)

(b)

Figure 6. Setup for fabrication of the MLAHOE, (a) the first step, and (b) the second step.

In the second step, the experimental setup is shown in Figure $6 \mathrm{~b}$. The laser beam is divided into two beams by a BS. One of the laser beams is expanded by a SF and a CL, then the laser beam incident into the micro-lens array with the angle $\theta_{4}=50^{\circ}$ forms spherical wave array II. Another laser beam passes through the DL to form spherical wave II. The angle of spherical wave II incident into the holographic material is controlled to be $\theta_{5}=61^{\circ}$, and $\theta_{6}=81^{\circ}$. The interference occurs between the spherical waves I and spherical wavefront array I, and the interference fringes are recorded on the holographic plate. The holographic plate is post-processed to obtain the required MHOE. The size of the MHOE is $80 \mathrm{~mm} \times 80 \mathrm{~mm}$, and the diffraction efficiency and transmittance of the MHOE are greater than $85 \%$ and $80 \%$, respectively. The exposure time is controlled by the electronic shutters. The detailed parameters of the optical devices are shown in Table 1. 
Table 1. Parameters of the optical devices in our experiments.

\begin{tabular}{ccc}
\hline Components & Parameters & Values \\
\hline \multirow{2}{*}{ Green laser } & Power & $400 \mathrm{~mW}$ \\
& Wavelength & $532 \mathrm{~nm}$ \\
\hline Green sensitive photopolymer material & Thickness & $15 \mathrm{um}$ \\
& Resolution & $12000 \mathrm{line} / \mathrm{mm}$ \\
& Sensitivity & $10 \mathrm{~mJ} / \mathrm{cm}^{2}$ \\
& Averaged refractive index & 1.47 \\
\hline Micro-lens array & Pitch & $1 \mathrm{~mm}$ \\
& Focal length & $3.3 \mathrm{~mm}$ \\
\hline Beam splitter & Coupling ration & $1: 1$ \\
\hline \multirow{2}{*}{ Projector } & Model & $\mathrm{MX} 518 \mathrm{~F}$ \\
& Resolution & $1600 \times 1200$ \\
& Horizontal scanning frequency & $102 \mathrm{KHz}$ \\
& Vertical scanning frequency & $102 \mathrm{~Hz}$ \\
\hline
\end{tabular}

In this paper, the MHOE is used as a projection screen, when the incident projection beam deviates greatly from the Bragg condition, the diffracted beam cannot be reconstructed and the 3D images also cannot be reconstructed. Therefore, the angular selectivity of the MHOE needs to be measured. The maximum deviated angle of the projection beam must be measured but the diffracted beam and 3D images cannot be reconstructed. The diffraction efficiency of the MHOE can be measured by the intensity of the transmitted and diffracted beam. The measurement formula of the diffraction efficiency can be expressed as [21]:

$$
\eta=\frac{I_{D}}{I_{T}+I_{D}}
$$

where $I_{D}$ and $I_{T}$ represent the intensity of the diffracted and transmitted beam, respectively. During the measurement, the incident angle of the reference beam is controlled by a rotation table, and the intensity of the diffracted beam of the MHOE is obtained by an optical power meter. The diffraction efficiency of the left and right view zones of the MHOE is measured, as shown in Figure 7. Through observation, when the incident angle of the reference beam is at $13^{\circ}$, the diffraction efficiency of the MHOE is less than $5 \%$. When the incident angle of the reference beam is between $-4.5^{\circ}$ and $4.5^{\circ}$, the diffraction efficiency of the MHOE is higher than $50 \%$. As shown in Figure 7, the maximum deviated angle of the MHOE is between $-5^{\circ}$ and $5^{\circ}$ in the left and right viewing zones.

The angle between the incident angle $\theta_{3}$ of projector I and the incident angle $\theta_{5}$ of projector II is much larger than the maximum deviated angle of the MHOE, that is $38^{\circ}>10^{\circ}$. The angle between the incident angle $\theta_{2}$ of projector I and the incident angle $\theta_{6}$ of projector II is much larger than the maximum deviated angle of the MHOE, that is $38^{\circ}>10^{\circ}$. Therefore, there is no crosstalk between the left and right viewing zones.

The projector (BenQ MX518F) is used to ensure the diffraction efficiencies of the MHOE. The horizontal scanning frequency is $102 \mathrm{KHz}$, and the vertical scanning frequency is $120 \mathrm{~Hz}$. The resolution of the projector is $1600 \times 1200$. The detailed parameters of the projector are shown in Table 1. Projection beam I is projected onto the MHOE with a pair of angles $\theta_{2}=61^{\circ}$ and $\theta_{3}=81^{\circ}$, which are the same as in the first step. Then, spherical wavefront array I is reconstructed in the left direction, as shown in Figure 8a. Projection beam II is projected onto the MHOE with a pair of angles $\theta_{5}=81^{\circ}$ and $\theta_{6}=61^{\circ}$, which are the same as in the second step. Then, spherical wavefront array II is reconstructed in the right direction, as shown in Figure 8b. 


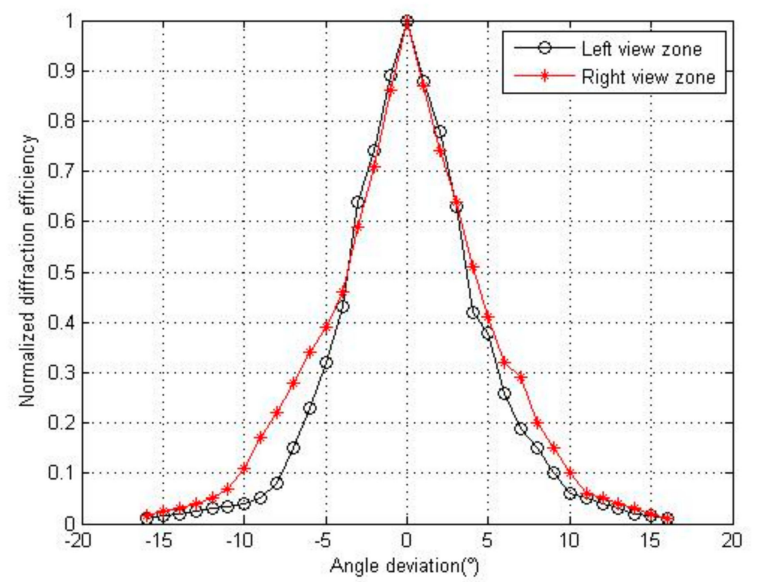

Figure 7. Diffraction efficiency of the left and right viewing zone.

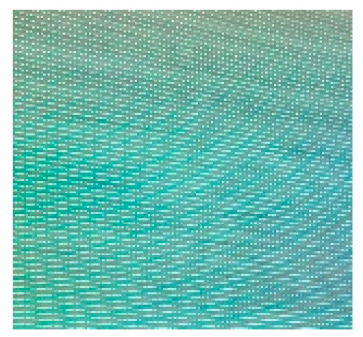

(a)

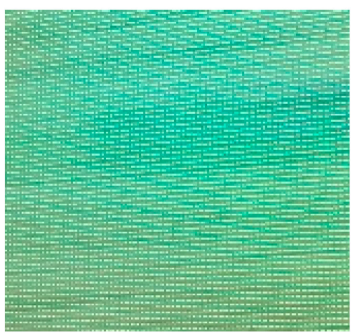

(b)

Figure 8. Reconstruction of the wavefronts of the lens array (a) in the left viewing zone, and (b) in the right viewing zone.

\subsection{Experiments for Displaying Images on the Proposed Dual-View 3D Display}

The projector is used in the experiment for displaying images. According to the incident angle requirement of the $\mathrm{MHOE}$, projector I is placed on the left, and projection beam I is incident into the MHOE with a pair of angles $\theta_{2}=61^{\circ}$ and $\theta_{3}=81^{\circ}$. Projector II is placed on the right, and projection beam II is incident onto the MHOE with a pair of angles $\theta_{5}=81^{\circ}$ and $\theta_{6}=61^{\circ}$. Therefore, projection beams I and II are symmetrical to each other.

Figure 9 shows the experiment setup for the dual-view 3D display. In order to satisfy the incident angle about the MHOE, the two projectors are placed vertically, and the projection lens is positioned inside. The distance between the projection lenses of the projectors and the MHOE is $31 \mathrm{~cm}$. Projectors I and II are $20 \mathrm{~cm}$ apart from each other in the horizontal direction. The projection lenses of projectors I and II are both $21 \mathrm{~cm}$ away from the optical table. The height of the MHOE is $19.5 \mathrm{~cm}$ from the optical table. The distance between the MHOE and the car model is $3 \mathrm{~cm}$. The projectors are used to project the EIAs onto the MHOE. The projection beams of the projectors that meet the Bragg-match are directly projected onto the MHOE. The MHOE is used as a screen of the dual-view 3D display. Projector I projects EIA I as projection beam I, with a pair of angles $\theta_{2}=61^{\circ}$ and $\theta_{3}=81^{\circ}$ onto the MHOE. Then, $3 \mathrm{D}$ image I is reconstructed in the left viewing zone. Projector II projects EIA II as projection beam II, with a pair of angles $\theta_{5}=61^{\circ}$ and $\theta_{6}=81^{\circ}$ onto the MHOE. Then, 3D image II is reconstructed in the right viewing zone. In this paper, the pith of the elemental lens in the micro-lens array is $1 \mathrm{~mm}$ and the focal length is $3.3 \mathrm{~mm}$. According to Equations (3) and (4), the viewing angles of the left and right viewing zone are $\Omega_{1}=10.2^{\circ}$ and $\Omega_{2}=10.2^{\circ}$, respectively. According to the parameters of the micro-lens array, the viewing angle of the conventional integral imaging is about $12^{\circ}$. Therefore, the viewing angles in the dual viewing zones are almost the same as the conventional one. 


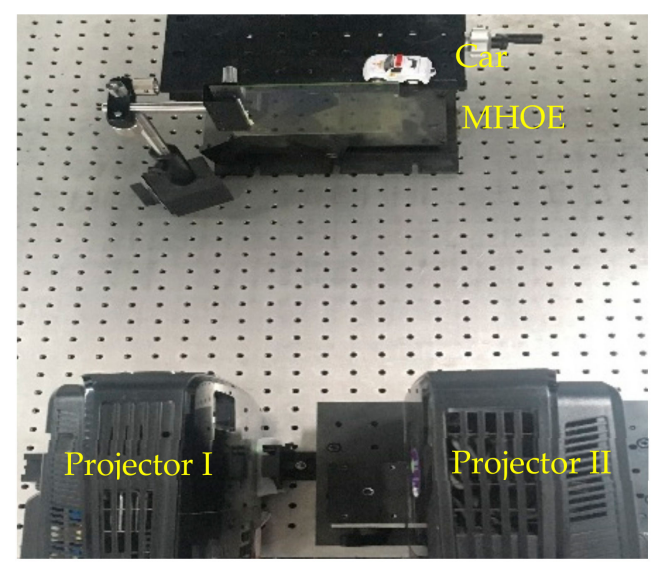

Figure 9. Experiment setup for the dual-view 3D display.

EIAs I and II are shown in Figure 10a,b, respectively. Each EIA consists of $70 \times 70$ image elements. EIA I is generated by a computer and consists of the characters " 3 " and " $\mathrm{D}$ ", in which " 3 " is located at $-15 \mathrm{~mm}$, and " $\mathrm{D}$ " is located at $+15 \mathrm{~mm}$. EIA II is also generated by a computer and consists of the characters " $A$ " and " $B$ ", in which " $A$ " is located at $-15 \mathrm{~mm}$, and " $B$ " is located at $+15 \mathrm{~mm}$.

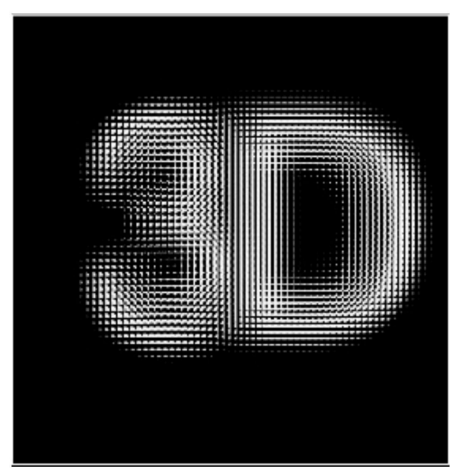

(a)

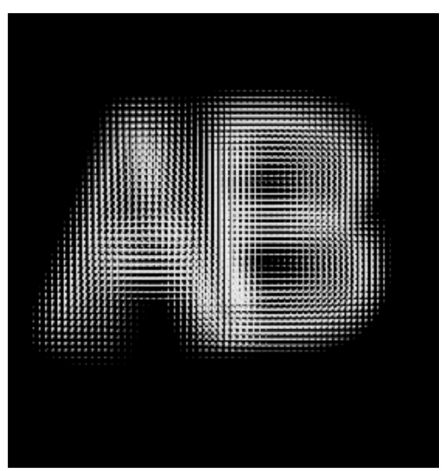

(b)

Figure 10. (a) EIA I and (b) EIA II.

3D image I reconstructed in the left view zone taken from the left, right, top and bottom directions are shown in Figure 11. We can see the horizontal parallax of the "3D" images from the left and right directions, and the vertical parallax of the " $3 \mathrm{D}^{\prime}$ images from the top and bottom directions. Reconstructed 3D image I has obvious horizontal and vertical parallaxes. Visualizations 1 and 2 clearly show the horizontal and vertical parallaxes between the " 3 ", " $\mathrm{D}$ " and the real object. The resolution of reconstructed 3D image I is almost the same as the conventional integral imaging 3D display, and 3D image I in the left view zone has high brightness and definition. The resolution of 3D image I is the same as the number of the image element $70 \times 70$ in EIA I.

Figure 12 shows 3D image II reconstructed in the right view zone taken from the left, right, top and bottom directions. We can see the horizontal parallax of the " $A B$ " images from the left and

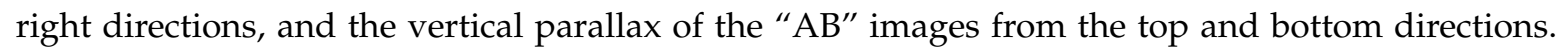
The reconstructed 3D image II has obvious horizontal and vertical parallaxes. Visualizations 2 and 3 clearly show the horizontal and vertical parallaxes between the " $\mathrm{A}$ ", " $\mathrm{B}$ " and the real object. The resolution of reconstructed 3D image II is also almost the same as the conventional integral imaging 3D display. The resolution of 3D image II is also the same as the number of the image element $70 \times 70$ in EIA II. The 3D images I and II have the same resolution, according to the parameters of the micro-lens array, the resolution of the conventional integral imaging is $70 \times 70$. Therefore, the resolution in the dual viewing zones are the same as the conventional one. 
It was observed that there is slight image crosstalk in the 3D image reconstructed in the left and right viewing zone. When projection beam I is projected onto the MHOE, the light produces diffuse reflection on the glass substrate of the $\mathrm{MHOE}$, and the diffuse light can produce slight crosstalk with reconstructed 3D image II in the right viewing zone. Reconstructed 3D image I in the left viewing zone has slight crosstalk, which is caused by the same reason as in the right viewing zone.
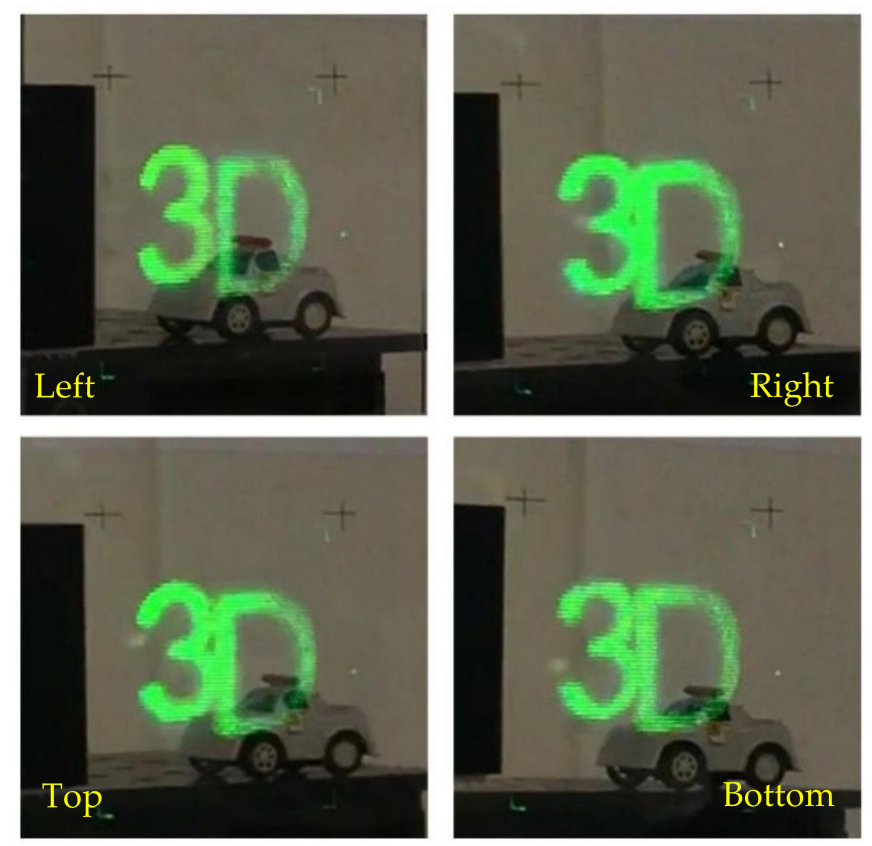

Figure 11. Experimental results in the left viewing zone (see Supplementary Materials visualization 1 and visualization 2), the parallax images in the left, right, top, and bottom four directions.
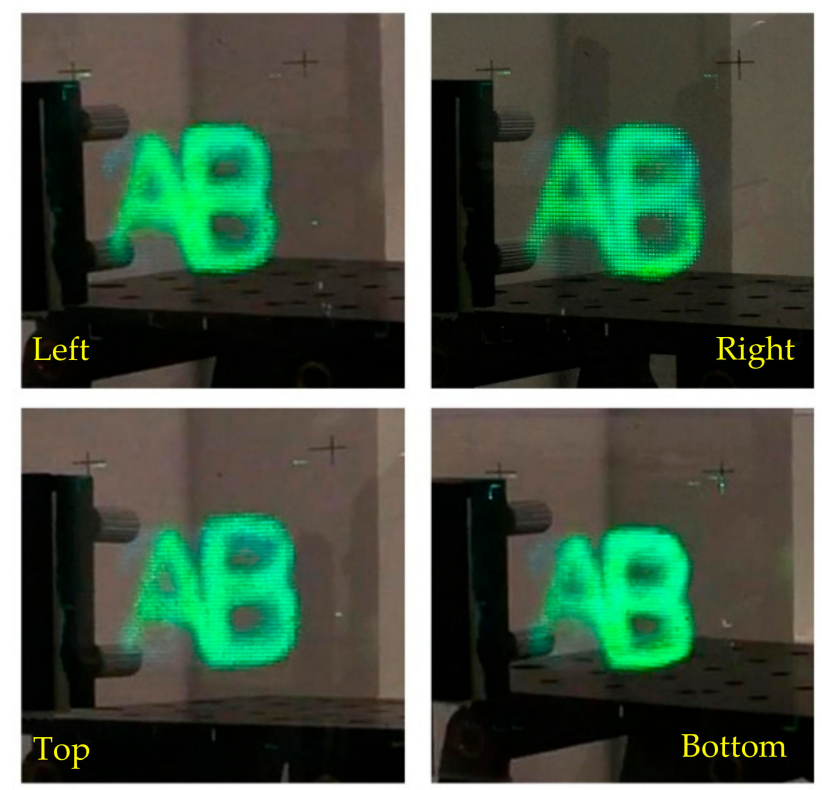

Figure 12. Experimental results in the right viewing zone (see Supplementary Materials visualization 2 and visualization 3), the parallax images in the left, right, top, and bottom four directions.

\section{Conclusions}

In this paper, we propose a dual-view 3D display. Two projectors are used to present different 3D images on the MHOE. Viewers can see the two different 3D images in the left and right viewing zones. The viewing angle and resolution of the reconstructed 3D images in the two viewing zones are almost 
the same as the conventional integral imaging 3D display. In addition, the fusion of 3D images with real objects is realized. It can be used for the car head up display and medical devices.

Supplementary Materials: The following are available online at http://www.mdpi.com/2076-3417/9/18/3852/s1.

Author Contributions: Conceptualization, Q.W. and D.L.; methodology, H.Z.; software, H.Z.; validation, Q.W., H.D.; formal analysis, H.Z.; investigation, M.H.; writing—original draft preparation, H.Z.; writing—review and editing, H.Z., Q.W., D.H., M.H., D.L.; visualization, H.Z.; funding acquisition, Q.W.

Funding: This work is supported by the National Key R\&D Program of China (2017YFB1002900) and National Natural Science Foundation of China (61535007 and 61775151).

Conflicts of Interest: The authors declare no conflict of interest.

\section{References}

1. Cui, J.P.; Li, Y.; Yan, J.; Cheng, H.C.; Wang, Q.H. Time-multiplexed dual-view display using a blue phase liquid crystal. J. Disp. Technol. 2013, 9, 87-90. [CrossRef]

2. Hsieh, C.T.; Shu, J.N.; Chen, H.T.; Huang, C.Y.; Tian, C.J.; Lin, C.H. Dual-view liquid crystal display fabricated by patterned electrodes. Opt. Express 2012, 20, 8641-8648. [CrossRef] [PubMed]

3. Zhao, Y.; Cao, L.; Zhang, H.; Kong, D.; Jin, G. Accurate calculation of computer-generated holograms using angular-spectrum layer-oriented method. Opt. Express 2015, 23, 25440-25449. [CrossRef] [PubMed]

4. Zhang, H.; Zhao, Y.; Cao, L.; Jin, G. Fully computed holographic stereogram based algorithm for computer-generated holograms with accurate depth cues. Opt. Express 2015, 23, 3901-3913. [CrossRef] [PubMed]

5. Gao, Q.; Liu, J.; Han, J.; Li, X. Monocular 3D see-through head-mounted display via complex amplitude modulation. Opt. Express 2016, 24, 17372-17383. [CrossRef] [PubMed]

6. Sang, X.; Fan, F.C.; Jiang, C.C.; Choi, S.; Dou, W.; Yu, C. Demonstration of a large-size real-time full-color three-dimensional display. Opt. Lett. 2009, 34, 3803-3805. [CrossRef] [PubMed]

7. Yu, C.; Yuan, J.; Fan, F.C.; Jiang, C.C.; Choi, S.; Sang, X. The modulation function and realizing method of holographic functional screen. Opt. Express 2010, 18, 27820-27826. [CrossRef] [PubMed]

8. Lee, H.H.; Huang, P.J.; Wu, J.Y.; Hsieh, P.Y.; Huang, Y.P. A 2D/3D hybrid integral imaging display by using fast switchable hexagonal liquid crystal lens array. In Proceedings of the Three-dimensional Imaging, Visualization, \& Display. International Society for Optics and Photonics, Anaheim, CA, USA, 10 May 2017; Volume 10219, pp. 1021910-1021916.

9. Oi, R.; Chou, P.Y.; Jackin, B.J.; Wakunami, K.; Ichihashi, Y.; Okui, M.; Huang, Y.P.; Yamamoto, K. Three-dimensional reflection screens fabricated by holographic wavefront printer. Opt. Eng. 2018, 57, 61605-61612.

10. Patel, S.K.; Cao, J.; Lippert, A.R. A volumetric three-dimensional digital light photoactivatable dye display. Nat. Commun. 2017, 8, 15239-15246. [CrossRef] [PubMed]

11. Kota, K.; Satoshi, H.; Yoshio, H. Volumetric bubble display. Optica 2017, 4, 298-302.

12. Wang, $\mathrm{X}$.; Hua, $\mathrm{H}$. Theoretical analysis for integral imaging performance based on microscanning of a microlens array. Opt. Lett. 2008, 33, 449-451. [CrossRef] [PubMed]

13. Javidi, B.; Hua, H. A 3d integral imaging optical see-through head-mounted display. Opt. Express 2014, 22, 13484-13491.

14. Zhang, H.L.; Deng, H.; Yu, W.T.; He, M.Y.; Li, D.H.; Wang, Q.H. Tabletop augmented reality 3D display system based on integral imaging. J. Opt. Soc. Am. B 2017, 34, 16. [CrossRef]

15. He, M.Y.; Zhang, H.L.; Deng, H.; Li, X.W.; Wang, Q.H. Dual-view-zone tabletop 3D display system based on integral imaging. Appl. Opt. 2018, 57, 952-958. [CrossRef] [PubMed]

16. Wu, F.; Deng, H.; Luo, C.G.; Li, D.H.; Wang, Q.H. Dual-view integral imaging three-dimensional display. Appl. Opt. 2013, 52, 4911-4914. [CrossRef] [PubMed]

17. Wu, F.; Wang, Q.H.; Luo, C.G.; Li, D.H.; Deng, H. Dual-view integral imaging 3D display using polarizer parallax barriers. Appl. Opt. 2014, 53, 2037-2039. [CrossRef] [PubMed]

18. Wang, Q.H.; Ji, C.C.; Li, L.; Deng, H. Dual-view integral imaging 3D display by using orthogonal polarizer array and polarization switcher. Opt. Express 2016, 24, 9-16. [CrossRef] [PubMed] 
19. Jeong, J.; Lee, C.K.; Hong, K.; Yeom, J.; Lee, B. Projection-type dual-view three-dimensional display system based on integral imaging. Appl. Opt. 2014, 53, 12-18. [CrossRef] [PubMed]

20. Li, G.; Lee, D.; Jeong, Y.; Lee, B. Fourier holographic display for augmented reality using holographic optical element. Disp. Technol. Int. Soc. Opt. Photonics 2016, 9770, 97700D.

21. Tao, S.Q.; Wang, D.Y.; Qing, J.Z.; Yuan, Q. Optical Hologram Storage; Beijing University of Technology Press: Beijing, China, 1998. 